\title{
Urban adaptation post-earthquake
}

\author{
C. Zanirato \& M. Contini \\ DiDA, Department of Architecture, Florence University, Italy
}

\begin{abstract}
Cities can grow only when something changes from within, by continuously updating with the times and through innovation. We're talking about a qualitative growth, the only thing able to give the city a higher status. The postearthquake reconstruction plan for a town or city hit hard, like Mirandola, is a great opportunity to reflect on such dynamics. Comparing the historical map of the fortified city in the Renaissance with the recent expansion, it is clear that the forma Urbis is clearly recognizable, it does not match any anything else: a precise conformation that has been followed by only one augmentative process. No longer able to think in terms of an "accomplished" urban form, we can operate profitably in terms of systems, "conforming" urban systems to govern the transformation, the growth. The starting point was a system of slow mobility: creating, planning and completing pedestrian and cycle paths; modifying vehicular access, extending pedestrian areas and offering more public and private parking; conserving and safe-guarding public parks with appropriate facilities, piecing them together with the open spaces of the countryside and conforming new settlements according to urban design; and last but not least, the reconstruction of buildings damaged by the earthquake for a renewed urban setting. Even though it is difficult to think of a physical transformation of a city, it is possible to intervene in its functional form, relatively intangible, but nevertheless effective. The earthquake of 2012 had a strong impact on the urban fabric of the town, altering the relationship between "full" and "empty": the ruins have left a glimpse of the unexpected relationship between these two elements, and uninhabited areas have acquired a strategic value for the relocation of parts of the city.
\end{abstract}

Keywords: urban shape, reconfiguration of the town, rehabilitation postearthquake, urban accessibility, urban design. 


\section{Introduction}

Man creates shape and brings it to life through separation and union, and the shape can change into an image only if it succeeds in parting from the background thanks to its contour, its limit. For this reason every town, just as every architecture, gets shape from the surrounding environment [1]. The ancient settlement of Mirandola found meaning in the contrast with the marshes which characterize the Po valley. Once the marshes were drained, the farmland which took their place irrevocably changed the original relationship and improved its dissolution into a borderless city that has become, in recent decades, the European capital of the largest biomedical district. The background of this city, once characterized by marshes, then by fields, more recently looks like a scattered collection of houses and factories, without a clear line or border.

Can the pre-existing urban voids, both physical and functional, and those caused by the earthquake, combined with urban regeneration, allow the restoration of fragments of a landscape believed to be lost? The earthquake, that struck Mirandola and the neighbouring areas in 2012, has not caused widespread destruction, like previous earthquakes in Aquila, Irpinia and Friuli, but it worked in a selective way. The sussultatory movement has mainly done more serious damage to the great historical and specialized buildings and much less to the houses: primarily churches and bell towers, but also castles and towers, theatres, town halls, schools, barns...... Therefore, the historical settlement of the rural landscape and the fundamentals of the urban landscape of many small cities are now missing. Nearly all the public places and buildings where citizens would meet up were destroyed, but also places where people perceived the major deficiencies in the functioning of the urban environment.

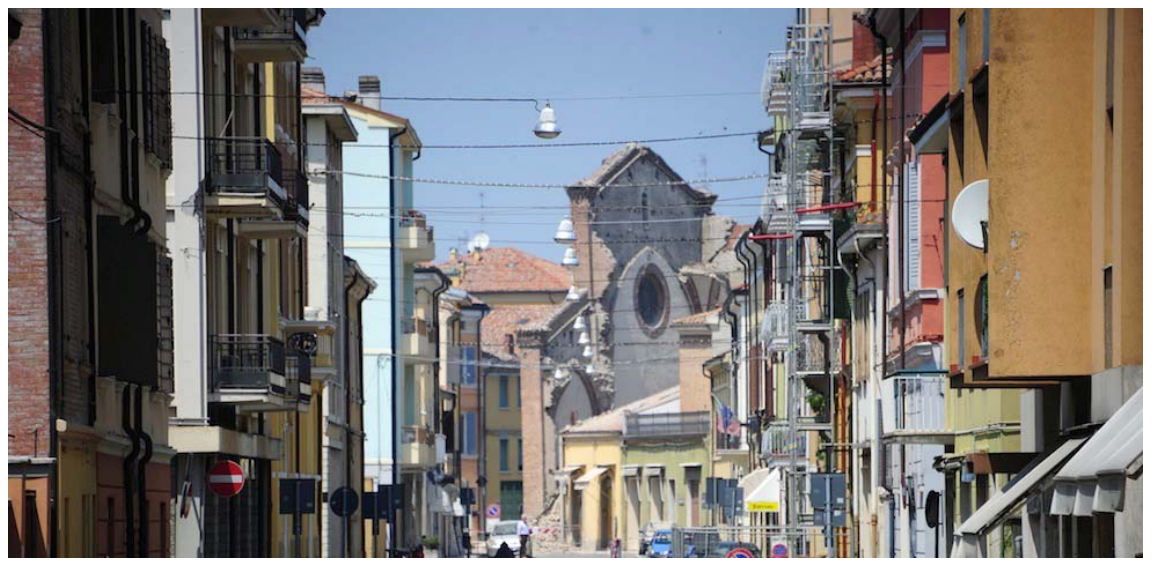

Figure 1: Localized collapse of buildings in Mirandola.

Sporadic and selective demolition will allow "surgical" repair interventions, otherwise impossible. Urban planning choices have pushed the first urgent interventions and the following reconstruction plans and moved towards a logic 
of substitution, migration of activities, re-localization: new schools built in the suburbs and old schools reconverted into city halls, old city halls will became libraries and museums....., several incongruous buildings will not be reconstructed and green and sporting areas will be used for new buildings. In this way cities obtain new structures and functions, making them possibly more sustainable. Several urban voids that were generated or that will be filled could be seen as unintentional "archaeological sites" in the urban layout and so allow a reinstatement of values and signs believed to be lost and rediscovered for new uses.

\section{Forma Urbis}

Occupation of a site and its transformation into a city are not enough to institute a tangible limit; that also needs a selection of thresholds to exist, to enable their crossing. In practice, a limit needs signs to define it, landscape or architectural elements to point out that something starts or ends, so that from a certain point and from a certain moment we enter something else, different from where we came from or where we left. Since birth limit reveals its fundamental characteristic: it points out the presence of a real or presumed difference. Therefore, through the recognition of a difference, drawing a border allows us to obtain a defined space, with its own rules and an autonomy also visible from the outside [2]. The threshold is the preferred place of crossing, it is where the transit connects and divides the spaces, without belonging to any, but introducing them, hence a neutral space, a spatial intersection. In this manner the threshold makes possible the coexistence of opposites and always alludes to a condition of passage, divides places, timings and events and at the same time combines what it separates. The urban composition of Mirandola, historically structured as an "accomplished" urban form, has seen for a long time, the presence of specific crossing points: pedestrian paths which used to "sink" in the marshy waters were then consolidated into streets and fortified city gates.

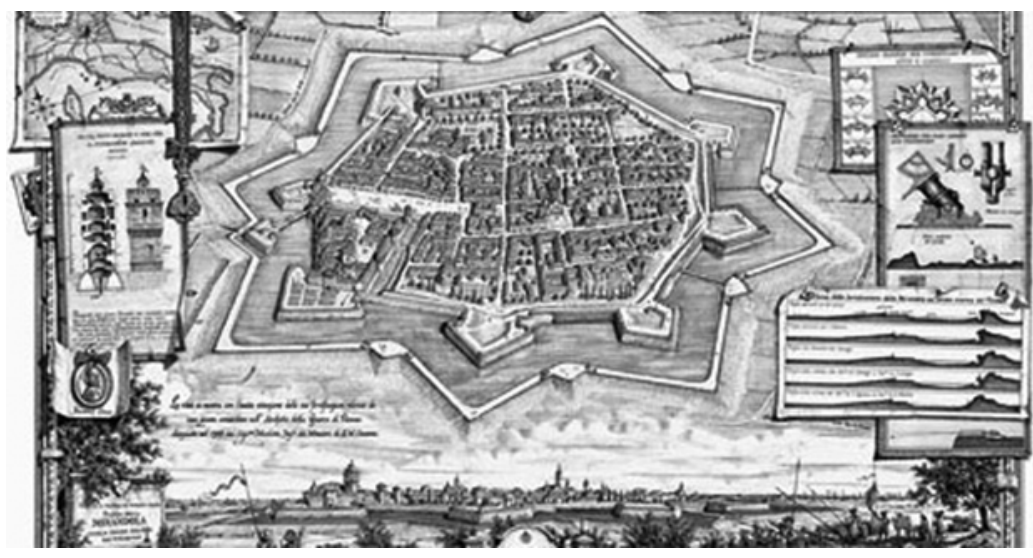

Figure 2: View of Mirandola in the 18th century. 
The walls surrounding Mirandola, although impressive and impenetrable, have never represented real barriers, even though their appearance would suggest so, as they do not create a break in the homogeneous space. The urban boundary, however, marks the difference, diversifies without any obstruction, suggests and emphasizes the precise points of passage, not necessarily just physical, as for example gates. Therefore, the urban border could represent at the same time a line of partition or connection according to its permeability, but the limit remains. The image of the Renaissance town of Mirandola, fortified with a "mathematical" polygonal "star", shows a consistent "thickness" of its environment, which has never been a simple line, but a layered area of transition between the urban settlement and its influent reflection in the territory. The physical structure of the urban boundary represented by the walls is typical of the old city, it is the element that makes the difference and is able to give finitude against the unlimited land [2].

Without evident limits, the possibility to cultivate and to build a space fades away, and therefore also the possibility to think in a different way, to be able to transform it and to expand it [2]. Traditional towns have always tried to highlight their borders, in a continuous search aimed at recognizing their physical status as an element of collective identity, their condition of stability and control. The main transformations of the limits and boundaries applied to the city and environment are related to the key evolutionary stages of human civilization: these transformations are the result of a defined cultural attitude, and for this they can be designed and can produce transformations in return. Edges, contours and borders are by their own nature irregular, permeable and movable and represent a defined exchange zone. The border creates the difference between the parts and therefore makes the exchange, the passage and the evasion possible [3].

Many cities were founded and based on the exchange that represents the vital character; from this arose the necessity to be preserved as open and mutable organisms in time and space, or else suffer an inevitable exhaustion and decadence. The city is not only a model in terms of shape, but also for development, and as a consequence shape must be the result of this evolutionary essence, moulded by the changes generated by the contrast between the wish for innovation and conservation. The history of cities is not a series of disconnected episodes, but it is a continuous and dynamic process, even in apparent discontinuity. The complex result of a stratification of events through which the urban organism becomes the demonstration of the existential ability that cities have always had for innovation and renovation, also bearing radical changes. The city, after the ' 800 crisis, has tried to enhance the architecture over the idea of delimitation: the walls evolved into boulevards, green belts and ring roads, then into structural interconnection and finally into "normative and IT networks", dissolving into the blurry reality we know.

\subsection{Conforming urban systems}

Over-stepping boundaries has always been a favourite in the modern world, to "go ahead" no matter what, tending to have nothing just "resting" on the border, 


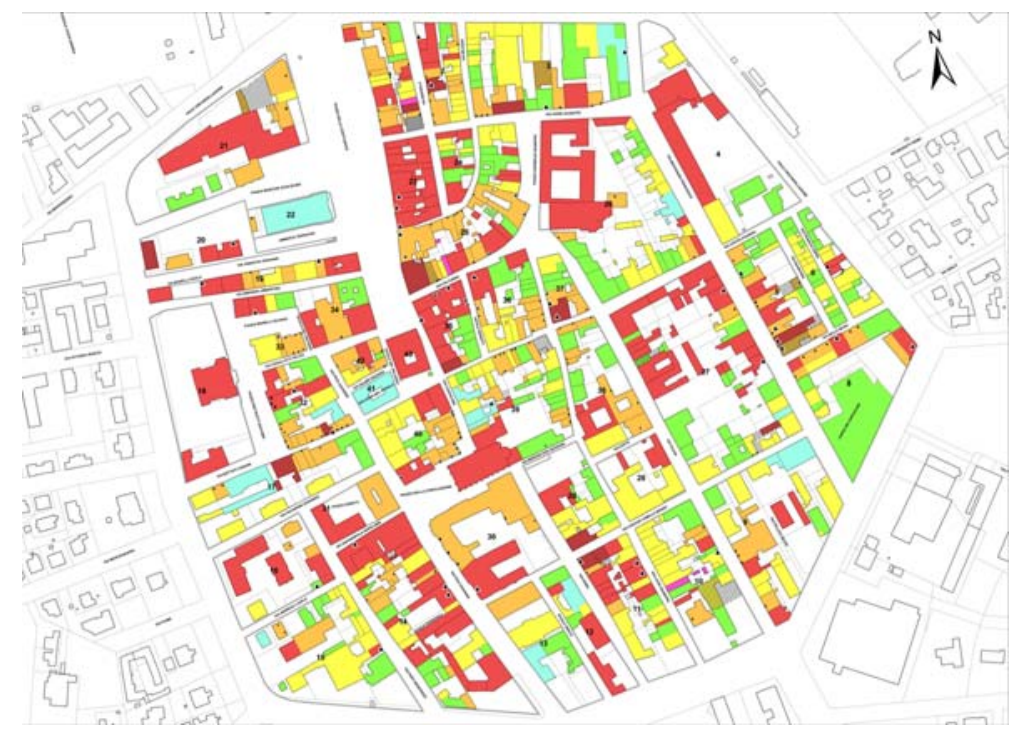

Figure 3: Damage in the centre, the most relevant in dark.

but everything spread everywhere, for everyone. It is ratified in the transition from a fenced-in city, a utopia, to a labyrinth city, Heterotopia, where onlycontinuous change is considered meaningful. After the city of Mirandola saw, first its "space" reduced, and then a complete demolition of its defensive walls and its embankments and ditches levelled off after an earthquake on 29 May 2012, only the "footprint" of its original shape was left. It had become a dormant figure, the once modern city replaced by a confused corporeality. Laying boundaries leads us to reflect on the referential cultural contexts that determine planning and design choices, already penetrated by a culture without limits, globalized, but still influenced and defined by certain local cultures, the result of old and new borders. It is for this reason that Mirandola has the opportunity to regain an equilibrium, recalling underlying innate boundaries, such as rites of passage, with new and updated roles.
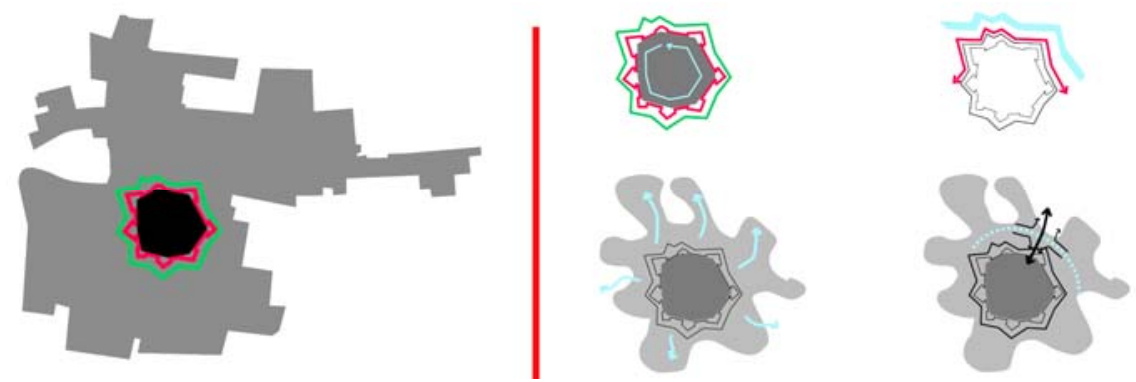

Figure 4: Border remodelling of the historical town. 
The northern part of this historic polygonal town, apart from the investment in amenities such as a rail system with station, bus station, a football stadium and other sports facilities and an office for the GIL (Fascist youth organization), has preserved an architectural rarefaction closer than anywhere else in the state before modern expansion. In this semi-circular perimeter, it is still possible to identify, albeit latently, the existence of that space of transition that for many centuries the city has been blessed with. There not only exists an outline of the original Renaissance city-fortress walls, but also moats and embankment. Parts of the castle fort and the Baroque Church of the Gesù (which is still completely intact) can be used as paths and guidelines in the planning, redesigning and restoring, integrating it even more with the town. The transformation of this quadrant of the city, therefore, by means of reshaping the space with or without man-made buildings, is an attempt to get back in touch with its historical past, building a space of transition. The project, as a whole, will have the urban model as its main goal, every part conforming, and having an organic, broad and open vision.
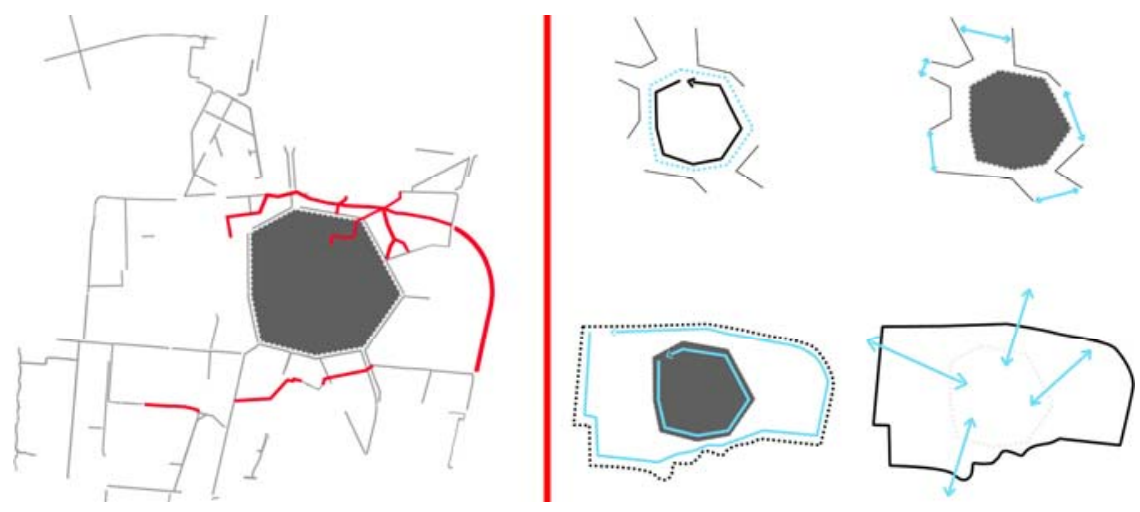

Figure 5: Structure of the suburban cycling paths.

\subsection{Slow mobility}

An extensive network of bike paths (already existing or underway) can be found accompanying the ring-road which circulates the historical town, like a blood vessel that, without penetrating or interfering with the existing life or physical structure, is simply an innervating part of the modern non-homogeneous town. A mobility such as this is an undeniable resource that shouldn't be ignored, but accompanied and assisted in its growth and urban development. The conformation of these paths needs to be implemented with a "target" of systematizing everything, with some added "seams" in order to obtain a logical "outline", which is still incomplete.

The creation of a second ring on the already existing ring-road could connect the peripheral parts of the first expansion of the city, where many of the more important public facilities are at present (the reconstruction plan may propose them to be transferred), effectively shortening commuting distance and reducing 
congestion in the innermost circuit around the historic structures. The routes proposed are planned for the north and west of the town centre (modelled on the remains of the earthquake-struck railway track), making their way across the open spaces and public green areas (following, as far as possible, the outline of the outer perimeter of the ancient moat's embankments to the north). In the southeast quadrant, only three connection interventions are needed. With these two closed circuits, the result would be a strengthened radial system, capable of penetrating the solidity of the historic city at strategic points, diffusing into the depths of urban sprawl and into productive areas, to go on to "disperse" into the surrounding countryside. The central Piazza Garibaldi is where the radial north-eastern bicycle path would finish. It will go beyond the historical perimeter, following the trail of a little water canal (partially revived), that leads to the hamlet of Quarantoli, ever mindful of the ancient moats and marshes of the past.
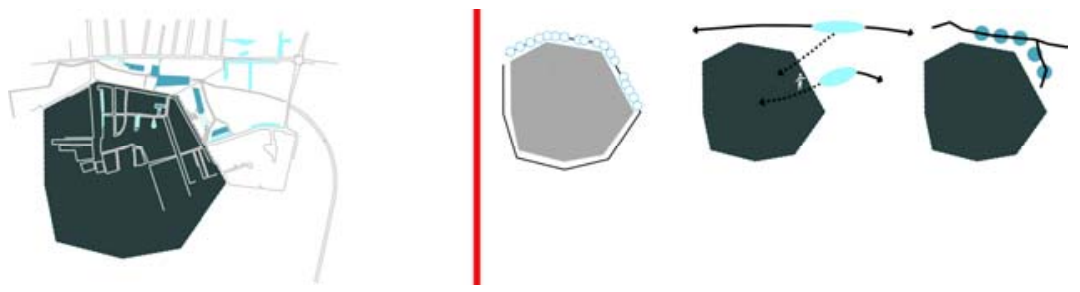

Figure 6: Structure of the suburban parking facilities.

\subsection{Fast mobility}

The traffic in Mirandola has already been effectively kept under control for some time now with a speed limit corresponding to the streets of the city and the implementation of a one-way traffic system. These initiatives were to avoid traffic congestion and to minimize unnecessary obtrusion in the historical centre.

In addition to these efforts already in place, a parking ban has been proposed, as far as possible, on stopping and long-term stays along the streets and roads in and around the town centre. This is to hopefully improve traffic flow further and, above all, to grant the necessary road space to reconfigure and facilitate the realization of pedestrian footpaths and bike paths, resulting in a true boulevard. Car parks, public and private, will, therefore, be divided into three distinct categories: underground, surface, covered and / or multi storey.

To cater for the need for post-earthquake construction in the northeast quadrant, mainly for trade and habitation (28 shops, 94 houses), the lower part of the new buildings will be designed to offer private owners/residents large underground long-stay car parks with a small portion for public use: for surplus demand, designated parking spaces and car parks will be provided on roads outside the town centre (a total of 450 parking spaces, of which 150 will be public). The construction of two covered car parks has been proposed for (daily) short-stay parking: in front of and in the proximity of the old stadium (with entry/exit from/to the ring-road). Constructing another floor above the covered 
playing field would provide 20 vehicle/car parking spaces and 30 motorbike parking spaces; another car park will be located inside the building of the locomotive depot, west of the station, with an automatic parking system (36 parking spaces).

For short-stay parking, the allocation of parking areas along the roads outside the city centre is expected to increase by an extra 100 parking spaces. (With 150 existing parking spaces already confirmed, there will be a total of 250.)

With such a reconfiguration of the amount of available parking for vehicles (much more than that resulting from other new interventions) differentiated in categories, we will be able to eliminate all parked vehicles along the streets in the town centre, enabling visitors and tourists to stop the car as near as possible to the boundary of the historical polygon, allowing them immediate access to the pedestrian and bike path routes to take them to the centre. This strategy can be extended to the whole urban area with a homogeneous and effective result.

\subsection{Pedestrianization}

When designing and providing public spaces, special attention to pedestrian areas is vital. The localization strategy is to mark out pedestrian walkways in the town streets at some transition points, between a dense inner and a more open outer place. It is the distinctive traits of some buildings that make these places stand out, the excellence of architecture scattered along the perimeter of the ring road (the castle, GIL, the railway station, the stadium etc.). These landmarks break up the continuity of the town streets, providing ideal "bridges" between one part and another, slowing things down, providing an experience and expanding the mind and vision. In these stretches the road meets the proportions of pedestrian paths, assuming different materiality, clearly indicating that it belongs. Similarly, other "emerging" surfaces present themselves around the churches, in front of the new developments and reconsigned historical changes. However, the designated spaces for pedestrians will be found not only on ground level, but also spread over raised areas, with the Bastione-Stazione walkway and the raised floor of the football field turned into a games and open-air show arena, which will be able to be used for other social/community events. This network of "piazzas" (or market squares/plazas) is not only for pedestrians but also entwined with the cycle paths.
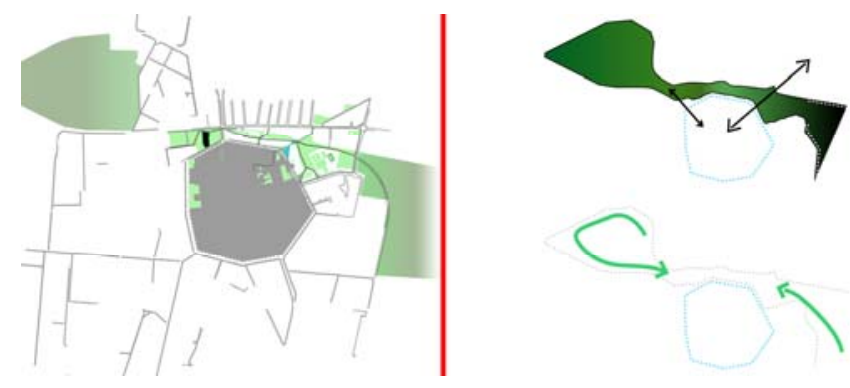

Figure 7: Restoration of partial relationship between old town and countryside. 


\subsection{The green structure}

The quadrant immediately north of the historic town centre has been kept, unlike the others, quite untouched by buildings, mainly due to the railway tracks that were the initial "protective" barrier, favouring the presence and construction of sports facilities; and also thanks to the important presence of the Castle, standing like a guardian, playing a big part in the discouragement of new construction the only exception being the building of GIL. Therefore, Mirandola inherits a concentration of space that has very few buildings, a green area sheltered from the northern ring road, a potential green band in the west, semi-engulfed by agriculture to the north and blending with the countryside to the east.

Public green areas are extremely important and need to be preserved and valued. In Mirandola's case, not only does it connect its historical core with the natural countryside, but it also comes between modern expansion, evoking the original full/empty balance in urban formalization. This is the reason why it is so important to support and safeguard the continuity of green spaces, placing new buildings in respect to pedestrian crossings, integrating vegetation as much as possible with the architecture, and planning roof gardens, patios and terraces for almost every new housing area. As well as this green area, there is also "peripheral" vegetation which penetrates the historical city; the peripheral garden of "Gesù" (which can be entered from the roads and from the town centre), and courtyards.

Water plays an essential role in the up-keep of public green spaces. Water runs along the ring road, and a "controlled" stream will be restructured, mindful of the system of ditches of the past and the origins of the Terramare culture of these places. The proposed main pedestrian paths will be completely immersed in this extended system $\left(35,200 \mathrm{~m}^{2}\right.$ of public green just in the areas concerned in the modification), adopting the linearity, and together they will form the planning foundation for the new part of the city.
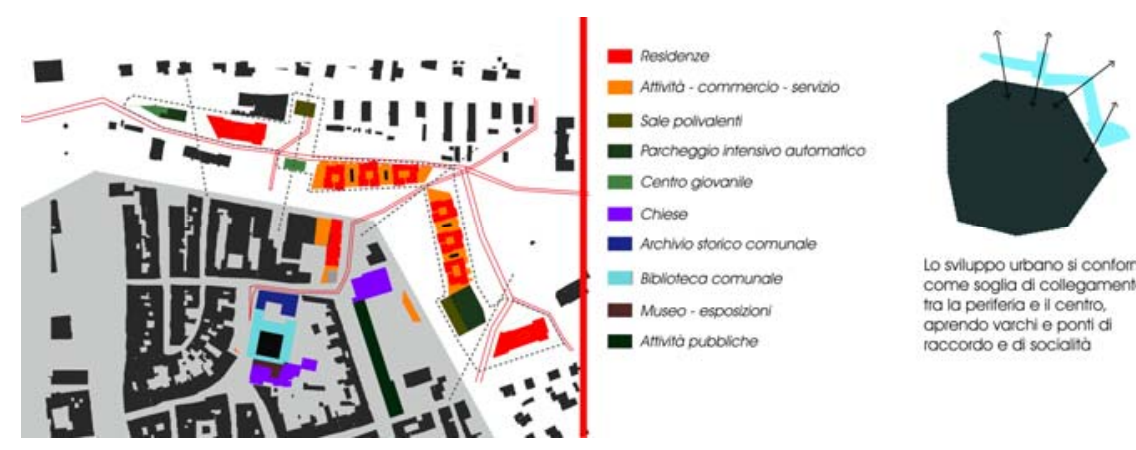

Lo sviluppo urbano si contorma come soglia di collegamento tra la periferia e il centro, aprendo varchi e ponti di raccordo e di socialita

Figure 8: Composition of new settlements and re-qualification. 


\subsection{Conforming settlements}

In distributing the activities, new roles and functions of the project, our goal is to mix urban presence, working with stratification and hybrids, physical and temporal, and as far as possible, with an unbroken continuity between them. The new architectural buildings have been designed following an ideal linearity that respects the layout of the ancient fortress and defensive moats which have long characterized the urban image of Mirandola, absorbing their pre-existence into the project designs, refunctionalized.

The best examples are two buildings which resemble the Bastione del Gesù (The Baroque Church of the Gesù). They define the doorway of the radial guideline for Quarantoli: a linear platform evoking the military urban architecture of the ancient embankment of the town, emptied of replenished earth, and in part "thrown aside", but cleverly reserved to build a raised shopping centre and services to support two-storey residential blocks.

The two sides of this segment will be juxtaposed. On one side stands the former Stadium, with the plane of the field raised from a share of the embankment. This is a space for games and entertainment, integrated into the covered platform below which can accommodate cultural, recreational and social club activities and events, strategically positioned next to the new covered public car park. On the other side stands the building of the former train station, adapted to accommodate other recreational activities - social and catering - with a boardwalk which will hook to the "bank", directed towards the former power plant, landing on a terrace. This technological building will be able to accommodate two multi-purpose rooms for shows and events of various natures, going on to building a cultural centre with underground parking and a public plaza, equipped and partly covered, to extend the planned activities outdoors. Slightly separated from these two polarities to the extreme west and south-east, there is just as much intensive residential construction planned, with a green area raised and beneath will be exclusive parking that will have another two doorways to the internal urban reconfiguration.

On completion of this, the transformation of the locomotive depot will be put into action. The depot shelter will house an automatic intensive public car park and the annex building will be redeployed for youth activities, with an expansion to create a local hangout. Naturally, all the buildings described here will also be well-connected with the two paths, pedestrian and cycle (annular and radial).

\section{Conclusions}

The final result of this design system is the restoration of a large area sandwiched between the historical city and its first expansion, which will be reorganized into a functional urban area, enhancing the historic core, and recovering a lost relationship with its interface immediately outside. Signs of the past too hastily eliminated or forgotten will start to emerge. A significant relationship will emerge between public parks and the city, implementing all possible systems and accessibility, finely tuned with the needs of everyone and 
everything. This is an example of a "relational" design, sensitive to place and the ever-changing times of town and city life, sustainable, as a true "interpreter".

Triggering a reversal of the value of full and empty spaces, inside and the outside the city, you can rethink the urban organism in a truly new way, as a "smart city" [4]. A resource as precious as this can truly represent a rebirth for a disaster-hit town or city, not only in a physical and economic sense, but also culturally.

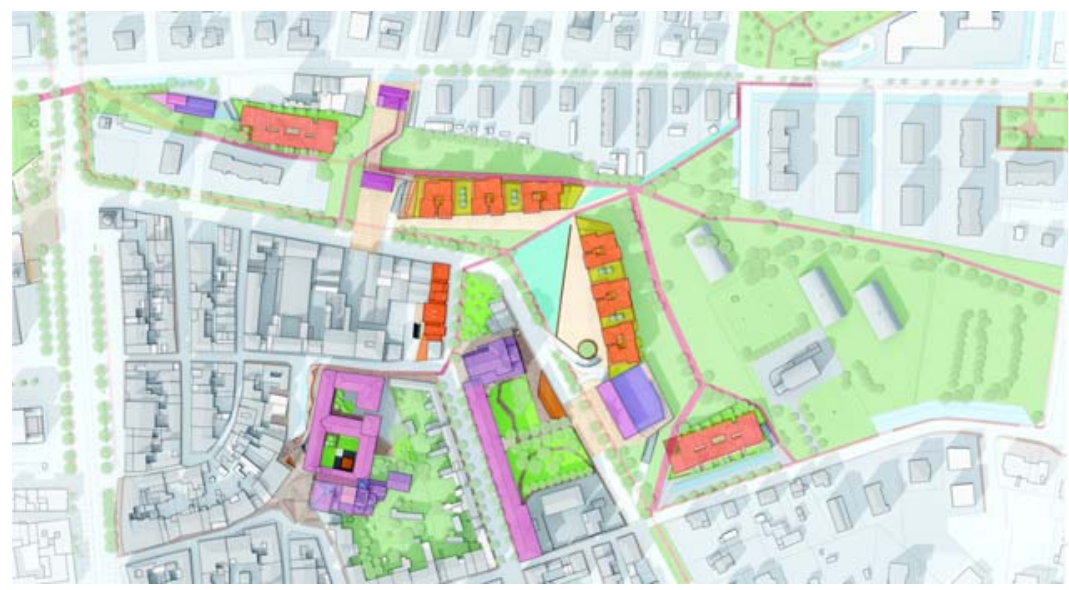

Figure 9: Masterplan of the north-east area of Mirandola.

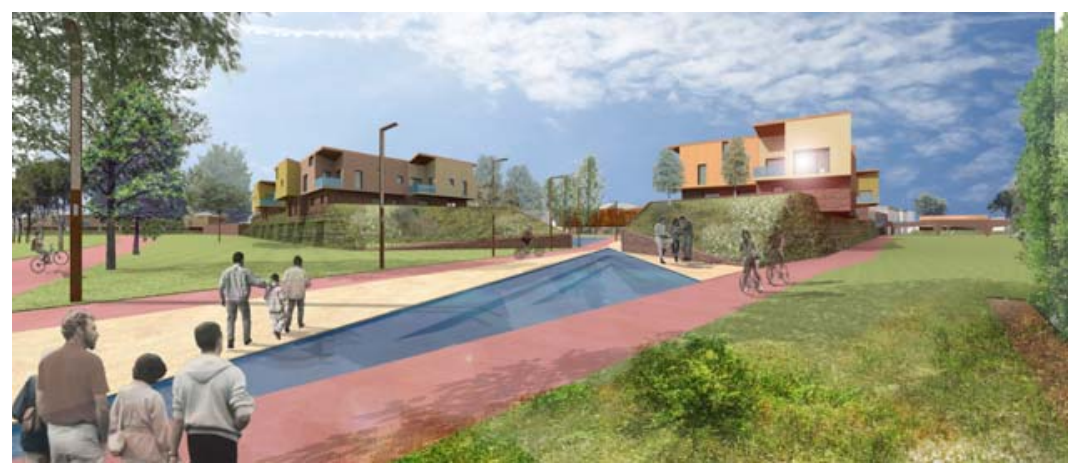

Figure 10: New access gate to the historical city of Mirandola.

\section{References}

[1] Lynch, K., L'immagine della città, Marsilio, Venezia, pp. 28-29, 2006.

[2] Zanini, P., I significati del confine, Mondadori, Milano, pp. 8-14, 1997.

[3] Albrecht, B. \& Benevolo, L., I confini del paesaggio, Laterza, Bari, p. 33, 1994.

[4] Landry, C., City Making, Codice Edizioni, Torino, pp. 396-397, 2006. 